\title{
Lusotopie
}

Recherches politiques internationales sur les espaces

issus de l'histoire et de la colonisation portugaises

$\mathrm{XV}(1) \mid 2008$

Indiens du Mozambique et d'Afrique orientale

\section{Les communautés indiennes au Burundi sous les colonisations allemande et belge}

Jean-Pierre Chrétien

\section{(2) OpenEdition \\ Journals}

Édition électronique

URL : http://journals.openedition.org/lusotopie/828

ISSN : 1768-3084

Éditeur :

Association des rechercheurs de la revue Lusotopie, Brill, Karthala

Édition imprimée

Date de publication : 30 juin 2008

Pagination : 161-173

ISSN : 1257-0273

Référence électronique

Jean-Pierre Chrétien, « Les communautés indiennes au Burundi sous les colonisations allemande et belge », Lusotopie [En ligne], XV(1) | 2008, mis en ligne le 09 mars 2016, consulté le 20 avril 2019. URL http://journals.openedition.org/lusotopie/828 


\section{LES GOMMUNAUTÉS INDIENNES AU BURUNDI SOUS LES GOLONISATIONS ALLEMANDE ET BELGE}

Depuis le début du $\mathrm{xx}^{\mathrm{e}}$ siècle des commerçants indiens, venus de la côte swahilie, sont établis sur les rives du lac Tanganyika. Au Burundi, ils passent de vingt en 1914 sous l'administration allemande à près de cinq cents en 1946 sous la tutelle belge. Ces «Indiens », à majorité masculine, jeune et mobile, travaillent à leur compte ou pour des firmes installées dans l'Est africain britannique. Ils se regroupent en réseaux à la fois économiques, familiaux et religieux (hindous, musulmans chiites, notamment ismaéliens, sunnites, catholiques goanais), et entretiennent des rapports distants avec les Africains et ambigus avec les colonisateurs. Cette microhistoire démographique et sociale est éclairée par les archives coloniales.

\section{As comunidades indianas no Burundi sob a colonização alemã e belga}

Desde o início do século XX, alguns comerciantes, vindos da costa suaile, estabeleceram-se nas margens do lago Tanganyika. No Burundi, eles passam de vinte em 1914 sob a administração alemã para cerca de quinhentos em 1946 sob a tutela belga. Estes «Indianos », de maioria masculina, jovem e com uma grande mobilidade, trabalham por conta própria ou para empresas implantadas na África oriental britânica. Eles agrupam-se em redes ao mesmo tempo económicas, familiares e religiosas (hindus, muçulmanos chiitas, especialmente ismaelitas, sunitas, católicos goeses), mantêm relações distantes com os Africanos e ambíguas com os colonizadores. Esta micro-história demográfica e social está baseada nos arquivos coloniais.

\section{Indian Communities in Burundi under German and Belgian Colonization}

From the beginning of the 20th Century, Indian traders from the Swahili coast settled on the shores of Lake Tanganyika. In Burundi, their number grew from twenty in 1914 under the German administration, to almost five hundred in 1946 under the Belgians. These young, mobile, mostly male "Indians" were selfemployed or worked for firms based in British East Africa. They formed networks based on economic, family and religious ties (Hindus, Muslims, Shiites, in particular Ismailis, Sunnites, Goan Catholics) and had distant relations with the Africans and ambiguous ones with the colonisers. The colonial archives illustrate this demographic and social micro-history.

$\mathrm{L}$ e Burundi sera pris ici comme un exemple particulier de l'Afrique des Grands Lacs. Mais il est impossible d'y comprendre la présence indienne durant la première moitié $\mathrm{du} \mathrm{xx}^{\mathrm{e}}$ siècle sans prendre en considération ce qui se passe à la même époque au Rwanda, en Ouganda, au Congo et surtout dans la Tanzanie actuelle $^{1}$. Nous y ferons souvent allusion.

${ }^{1}$ Voir J.-P. Chrétien, L'Afrique des Grands lacs. Deux mille ans d'histoire, Paris, Flammarion, 2003 


\section{L'héritage des relations avec la côte orientale : mobilité et croissance}

La présence des Indiens est liée historiquement à la pénétration des commerçants de Zanzibar jusqu'aux rives du lac Tanganyika depuis le milieu du xix ${ }^{\mathrm{e}}$ siècle et ensuite (à partir des années 1890) à l'intégration de cette région à l'ensemble de l'Afrique orientale allemande (Deutsch-Ostafrika) qui a représenté une aire d'expansion de ce qu'on peut appeler la culture swahilie, portée par des Arabes, par des Africains gagnés à l'Islam et au modèle culturel des villes de la côte, et enfin par des Indiens. Il est inutile de rappeler ici l'importance du rôle commercial et financier de ces derniers dans le sultanat de Zanzibar². Dès la première moitié du $\mathrm{XIx}^{\mathrm{e}}$ siècle, un khoja (ismaélien), surnommé Musa Mzuri, tient une place importante à Tabora sur la route des caravanes. L'intégration du Burundi dans l'ensemble belge ${ }^{3}$ après la Première Guerre mondiale rompt dans une certaine mesure ce lien avec l'Est et rend la situation des «Asiatiques » plus marginale, mais les relations continuent comme on le verra. Contrairement au cas du Kenya ou de l'Afrique du sud, l'émigration indienne dans ces parages n'est donc pas un déplacement de main-d'œuvre pour des chantiers ou des plantations. C'est le prolongement durable de l'expansion indienne dans l'océan Indien et les pays riverains. La plupart viennent du Nord-Ouest de l'Inde britannique, notamment de Bombay et de l'actuel Pakistan. Ils étaient 9000 en Afrique orientale allemande en $1914^{4}$.

Les Indiens sont mentionnés dans les dossiers allemands de Dar-es-Salaam ${ }^{5}$ consacrés à la région des lacs et dans les archives des Résidences de l'Urundi et du Ruanda ${ }^{6}$ à partir des années 1905-1907, c'est-à-dire dès le début de la mise en place de ces structures administratives ${ }^{7}$. Il s'agit de quelques commerçants implantés essentiellement à Ujiji au bord du Tanganyika ou à Bukoba au bord du Victoria, et qui envoient des colporteurs dans l'intérieur du Rwanda et du Burundi. Dès 1905, une Societa coloniale italiana envoie de son côté un Indien au Nord-Est du Burundi ${ }^{8}$. Néanmoins la station militaire d'Usumbura ne signale pour l'année suivante que trois Indiens installés dans son ressort (regroupant encore en

[1e éd. : Aubier, 2000]. J. ILIfFe, A modern history of Tanganyika, Cambridge, Cambridge University Press, 1979.

${ }^{2}$ Voir A. SHERIFF, Slaves, spices and ivory in Zanzibar. Integration of an East African commercial empire into the world economy, 1770-1873, Londres, James Currey, 1987.

${ }^{3}$ Voir J. Gahama, Le Burundi sous administration belge. La période du Mandat, 1919-1939, Paris, Karthala, 2001, [1e éd. : 1983].

${ }^{4}$ Voir R. Tetzlaff, Koloniale Entwicklung und Ausbeutung. Wirtschafts- und Sozialgeschichte DeutschOstafrikas 1885-1914, Berlin, Duncker \& Humblot, 1970.

${ }_{5}^{5}$ Tanzania National Archives (TNA), fonds allemands.

${ }^{6}$ Archives africaines de Bruxelles (AAB), fonds allemands. Notamment les rapports annuels de la Résidence (Fahresberichte) de 1909 à 1914 (microfilm 160).

${ }^{7}$ Auparavant l'ensemble était géré par les officiers de la Station militaire allemande d'Usumbura depuis 1897.

${ }^{8}$ Jahresberichte, op. cit.: f. 41. 
1906 les deux futures Résidences du Ruanda et de l'Urundi) ayant des boutiques dans les postes riverains des lacs Tanganyika et Kivu. Et il ne s'agit que de Béloutches ${ }^{9}$. Cette catégorie qui sera généralement traitée à part dans les archives ultérieures est composée de petits commerçants qui ont tenté fortune dans l'intérieur du continent après avoir été initialement des mercenaires (originaires du Béloutchistan) au service du sultan de Zanzibar, puis au service des caravanes ${ }^{10}$. L'année suivante, en 1907, les Indiens sont toujours trois dans le seul Burundi: Nur Mohamed Kanji, un employé indien de la firme Nasor Virji et un des Béloutches de l'année précédente (tous à Usumbura) ${ }^{11}$. En 1909, ils sont 15, dont deux femmes (et un Béloutche); en 1910, ils sont 21, dont 3 femmes et 4 enfants ; en 1911, ils sont 22. En 1912, sur les treize commerçants étrangers à Usumbura, 8 sont indiens; en 1913, ils sont 6 sur 17. À partir de 1912 est aussi mentionné un Goanais. Au début de 1914, dans l'ensemble des postes allemands des rives du Tanganyika au Burundi, sont recensés 17 Indiens (trois autres venant de repartir $)^{12}$. Au Rwanda à la même époque les Indiens sont deux fois plus nombreux, 46 en $1912^{13}$. Ces effectifs restent donc extrêmement faibles, bien inférieurs à ceux de l'Ouganda sous protectorat britannique : à la veille de la Première Guerre mondiale ces derniers étaient cent fois plus nombreux. Nous en verrons les raisons.

Après la Première Guerre mondiale, Rwanda et Burundi sont administrativement unis dans le Territoire du Ruanda-Urundi, composé de deux Résidences ${ }^{14}$, confié à l'administration belge sous mandat de la Société des Nations $(\mathrm{SDN})^{15}$. Dès lors le nombre des Indiens croît progressivement, bien que les deux pays soient devenus comme une extension du Congo, coupés de l'Afrique orientale devenue totalement britannique ${ }^{16}$. Pour le seul territoire d'Usumbura (chef-lieu du Territoire), au nord-est du lac Tanganyika, qui en abrite le plus grand nombre, nous observons que cette population a d'abord triplé en dix ans, et qu'elle a ensuite été multipliée par cinq entre le lendemain de la grande crise des années 1930 et le lendemain de la Seconde Guerre mondiale. Mais les effectifs demeurent modestes (tableau I).

9 TNA, G1/25, f. 32.

${ }^{10}$ F. Bontinck, "Commentaire d'un passage de Livingstone, Last journal, ler juin 1872 », Bulletin des séances de l'Académie royale des sciences d'outre-mer (Bruxelles), 1974, 4: 573.

11 TNA, G1/25, f. 32, 83, 123 (listes de firmes en 1906, 1907, 1908).

12 Fahresberichte de la Résidence de l'Urundi, AAB, 48.

13 Fahresberichte de la Résidence du Ruanda.

${ }^{14}$ Ne pas confondre ce Territoire sous mandat dans son ensemble et la subdivision des deux Résidences en «territoires " gérés par des administrateurs.

15 Sous tutelle de l'ONU de 1946 à 1962, date de l'indépendance retrouvée.

${ }^{16}$ Le reste de l'ancien Deutsch-Ostafrika devient le Tanganyika Territory (Territoire du Tanganyika), également sous mandat de la SDN. 
Tabl. I. - Indiens À Usumbura. Indigateurs démographiQues (1924-1946)

\begin{tabular}{lccc}
\hline Année & Nombre total & Dont femmes & Dont enfants \\
\hline 1924 & 36 & 5 & 14 \\
1927 & 30 & 6 & 6 \\
1928 & 42 & 8 & 9 \\
1929 & 81 & 21 & 19 \\
1932 & 86 & 19 & 29 \\
1936 & 94 & 16 & 21 \\
1939 & 130 & 23 & 39 \\
1940 & 159 & & \\
1946 & 475 & 84 & 250 \\
\hline
\end{tabular}

Source: Rapports annuels des territoires de la Résidence d'Urundi, Usumbura 1924-1949, AAB, RU 149 et 152; Rumonge et Nyanza-Lac 1924-1936, AAB, RU 117-118; dossiers AAB, BUR 219 et 221 (Asiatiques, 1931 et 1939).

En dehors d'Usumbura, on trouve également des Indiens dans les territoires ${ }^{17}$ de Kitega, au centre du pays, et de Bururi, au sud (en fait sur les rives du Tanganyika, dans les centres de Rumonge et de Nyanza-Lac). Mais apparemment il n'y en a pas ailleurs, ni au centre ni à l'est (par exemple à Muramvya, Rutana ou Ruyigi).

C'est ainsi qu'en 1939, les Indiens sont donc au nombre de 46 à Kitega (dont 7 femmes et 27 enfants). Ils sont 16 à Bururi (13 à Rumonge et 3 à Nyanza-Lac, dont 2 femmes et 6 enfants). Dans cette région Sud, leur présence remontait à l'époque allemande. Dans les années 1924-1933, nous avions déjà ces chiffres oscillant entre la dizaine et la quinzaine.

À ces effectifs s'ajoutent à Usumbura quelques «Béloutches » et aussi quelques Goanais (en 1928 les premiers sont trois, les seconds sept).

Sur le plan démographique, on observe une certaine stabilisation de cette population: le nombre des femmes et des enfants nés sur place augmente. Les femmes indiennes (mariées ou veuves) sont 5 pour 17 hommes actifs en 1924, 21 pour 41 en 1929, 23 pour 68 en 1939, 84 pour 141 en 1946. Le ratio, parti de plus de trois hommes adultes pour une femme, passe donc à près de deux en 1929, mais remonte à près de trois dix ans après, pour atteindre 1,65 en 1946 . Néanmoins les concubinages avec des filles du pays sont rares (du moins ouvertement): 6 cas mentionnés pour 45 hommes en 1928.

En fait la jeunesse et la mobilité restent donc grandes. En 1946, $35 \%$ ont moins de 10 ans, $21 \%$ de 10 ans à 20 ans, $17 \%$ de 20 à 30 ans, $16 \%$ de 30 à 40 ans, $7 \%$ de 40 à 50 ans, $4 \% 50$ ans et plus. Donc plus de la moitié ont moins de 20 ans, un tiers est dans la force de l'âge. Un peu plus d'une génération après leur installation dans cette région d'Afrique, les Indiens gardent une pyramide de migrants récents, très déséquilibrée dans le ratio des sexes, très jeune, mais avec une allure fluctuante d'année en année qui reflète moins une courbe de natalité et de mortalité que le rythme aléatoire des allées et venues.

${ }^{17}$ Chaque Résidence est divisée en «territoires» sous la responsabilité d'un administrateur belge. 
Dans les années 1930 (en combinant les différentes listes de 1936 et de 1939), on ne relève que 21 enfants dont la naissance est déclarée à Usumbura sur une soixantaine d'enfants. Beaucoup sont nés au Tanganyika, à Dar-es-Salaam ou Kigoma (aux deux extrémités du chemin de fer d'accès au Burundi), d'autres à Kigali, Bagamoyo, Zanzibar, mais aussi au Kenya (Lamu, Mombasa, Nyeri), et même deux à Madagascar (dont un à Tuléar) et un à Mehasana au Gujerat. Dans l'intérieur du Burundi, à Kitega, au contraire la plupart des enfants sont nés sur place, comme si la sédentarisation y était plus poussée. Dans les années 1930 nous sommes donc globalement à une période d'immigration importante que reflète cette diversité des lieux de naissance. Nous n'avons malheureusement pas cette indication dans les listes de 1946.

On imagine que pour les adultes, les origines sont encore plus diverses et lointaines: dans les listes de 1936-1939, nous trouvons 69 mentions de naissances en Inde (notamment Bombay, Ahmedabad, Vanthali, Nandur, Tandor, Jamnagar, Rajkot, Cutch), les autres sont nés surtout en Tanzanie actuelle (Dar-Es-Salaam, Kigoma ou Ujiji, Bagamoyo, Tabora), mais aussi à Zanzibar ou à Lamu, un à Albertville (au Congo), un à Tuléar (Madagascar)... La filière de la fin du XIx ${ }^{\mathrm{e}}$ siècle et du début du $\mathrm{xx}^{\mathrm{e}}$ siècle - Zanzibar et l'axe allemand de Dar-es-Salaam au lac Tanganyika -, continue à marquer la logique de ce peuplement. Une recherche plus intensive sur ces listes traitées sur le mode prosopographique et complétées par des enquêtes orales, permettrait de reconstituer des biographies familiales, révélant sans doute des mobilités plus complexes encore, faites de départs et de retours. Nous reviendrons sur les logiques économiques et culturelles de cette situation.

\section{Les activités : les réseaux commerciaux}

C'est le commerce qui constitue le domaine d'activité essentiel de cette communauté au Burundi, de ses débuts à nos jours. Leur arrivée se dessine surtout à partir de 1908, quand le Congo, passé sous le contrôle de la Belgique, renonce en principe aux monopoles léopoldiens et permet de nouveau le commerce libre de l'ivoire et du caoutchouc. Ces marchands utilisent donc les postes des bords du Tanganyika (Usumbura, mais aussi Rumonge, Nyanza ${ }^{18} \ldots$...) pour organiser ce trafic avec la rive congolaise. Les firmes représentées ont en général leur siège à Ujiji $^{19}$. Mais à partir des années 1910 un autre axe se dessine, celui du commerce des peaux (de vaches et surtout de chèvres) en direction du lac Victoria et du chemin de fer britannique qui relie l'Ouganda à Mombasa depuis 1902: cette fois il s'agit de firmes ayant leur siège à Bukoba ou à Kigali, ce trafic s'organisant d'abord à travers le Rwanda. Ces commerçants recourent généralement à des intermédiaires locaux, rwandais, burundais, congolais, pour le colportage des étoffes en échange des peaux ou du caoutchouc. Ces colporteurs travaillent à crédit. De même, les agents indiens fonctionnent à crédit par rapport aux firmes dont ils dépendent. Par ailleurs, des marchands ouvrent près des postes allemands des

${ }_{18}$ Nom de Nyanza-Lac à l'époque allemande. Ce toponyme d'origine swahilie désigne d'ailleurs le lac!

${ }^{19}$ Avec parfois un siège central plus éloigné, à Bagamoyo par exemple. 
boutiques (duka en swahili) où on trouve des cotonnades, mais aussi un peu de tout : à la veille du départ des Allemands, elles existent à Usumbura, à Rumonge, à Nyanza, mais aussi dans l'intérieur, à Gitega, à Nyakazu (au sud-est du pays) et même (sous forme de colportage) au nord-est. À cette époque les négociants les plus importants représentés au Burundi sont Nasor Virji, Abdallah Alarakia, Dossa Thawer, Merali Remtulla, Ali Nur Mohamed Kanji, Laddar Sumar (tous implantés au préalable à Ujiji) et aussi Omar Abdulkarim (implanté à Kigali) et Walji Banji (implanté à Bukoba) ${ }^{20}$.

Sous l'administration belge, les boutiques en dur vont se multiplier dans les principaux centres, essentiellement à Usumbura, chef-lieu du territoire sous mandat, et à Kitega, chef-lieu de la Résidence. Les Indiens sont, comme auparavant, soit à leur compte, soit gérants d'une firme basée à l'extérieur, soit de simples employés (vendeurs, secrétaires, chauffeurs) de ces commerçants.

Par exemple en 1932, sur les 34 Indiens actifs en territoire d'Usumbura, 9 sont commerçants, 17 employés dans ces entreprises. Il s'y ajoute 4 transporteurs, un hôtelier, un coiffeur, un charpentier, un cordonnier.

En 1946, sur 120 Indiens actifs dans le même territoire, 61 se déclarent commerçants, 30 employés ou clercs dans des entreprises, 7 transporteurs ou chauffeurs. Il s'y ajoute deux hôteliers, un tailleur, un cordonnier, deux coiffeurs, trois mécaniciens, trois instituteurs et un «entrepreneur ». Les activités artisanales ou industrielles sont donc minimes.

Le tableau des entreprises indiennes (incluant Béloutches et Goanais) à Usumbura en 1936 se présente ainsi :

Tabl. II. - Entreprises indienNes À Usumbura (1936)

\begin{tabular}{|c|c|}
\hline Nom & Activité \\
\hline Ali Rawji & commerce de traite, 3 magasins à Usa \\
\hline Allibhay Panju & commerce général, 3 magasins \\
\hline Bahader bin Hussein (béloutche) & commerce de traite \\
\hline Gopalji Damji & Charpentier \\
\hline Gulam Ussein Jaffer & commerce de traite* \\
\hline Gulam Hussein Ladak & commerce de traite* \\
\hline Hasham Lalji Kandji & commerce de traite* \\
\hline Hederali Gulamaji Mawji & commerce de traite, rizerie, 3 magasins \\
\hline Hussein Ali Musaji et Wali Jina & transport automobile \\
\hline Juthalal Velji & commerce général \\
\hline Kothari et Metha sté & commerce général \\
\hline Kandji Virji & commerce de traite* \\
\hline Lalji Jessa Kalidas & transport automobile \\
\hline Mohamed Hussein Alidina & commerce de traite* \\
\hline Merali Jetha & commerce de traite* \\
\hline
\end{tabular}

${ }^{20}$ Jahresberichte de la Résidence d'Urundi (1909-1914), op. cit.; Handel, Gewerbe, Verkehr, Schiffahrt, 1907-1915, AAB, 47. 
Tabl. II (Cont.)

\begin{tabular}{|c|c|}
\hline Nom & Activité \\
\hline Nurali Ibrahim & commerce de traite* \\
\hline Omar Hassan & hôtelier pour indigènes et asiatiques \\
\hline Ramabhai Maganlal & coiffeur \\
\hline Samji Ranaji & commerce de traite* \\
\hline Tadjeen Hassam Ali & commerce de traite* \\
\hline Usumbura trading $\mathrm{Cy}$ & commerce de traite, peaux \\
\hline De Souza Anacleto & commerce général \\
\hline De Souza Francisco & cultures maraîchères et vivrières \\
\hline Fernandes Sebastien & tailleur \\
\hline
\end{tabular}

* Expression désignant le commerce des produits primaires locaux (peaux, vivres, café). Source: Rapports annuels du Territoire d'Usumbura, AAB, RU 149.

En 1956 on retrouve quelques-uns de ces noms, classés cette fois en Indiens et Pakistanais (et Portugais pour les Goanais) :

Tabl. III. - Entreprises indienNes À Usumbura (1956)

Indiens
Ali Rawji (succession)
Amiralli Mohamed Murji
Gorajia GT
Juthalal Velji
Kothari Mehta
Samji frères
Tarmohamed Turk
Pakistanais
Abdulhussein Ladha
Portugais
De Souza Anacleto

commerce général affaires commerciales transports commerce général commerce général garage restaurant, cinéma transport et traite commerce général

Source: Rapports annuels du Territoire d'Usumbura, AAB, RU 152.

La plupart des salariés travaillent eux-mêmes dans des entreprises indiennes, sauf quelques exceptions. Les auxiliaires des Européens se recrutent davantage chez des Africains, d'abord les Swahili ${ }^{21}$, puis de plus en plus des Burundais catholiques.

En fait, les Indiens constituent un monde qui traverse tant les frontières du Burundi que celles des domaines coloniaux, présents chez les Allemands naguère, et ensuite à la fois (les mêmes firmes parfois) chez les Anglais et les Belges. Ils constituent des réseaux mi-économiques, mi-familiaux, mi-confessionnels. Les carrières des salariés devraient être suivies, comme nous l'avons déjà dit, aux différents

${ }^{21}$ Sur les Swahili des rives du lac Tanganyika, voir A. Abel, Les Musulmans noirs du Maniema, Bruxelles, Centre pour l'étude des problèmes du monde musulman contemporain, 1960. 
âges de leur existence : ils peuvent être employés d'abord dans un pays, puis dans un autre grâce à ces réseaux de relations. Les crises amènent des groupes à se replier vers une zone qui semble plus favorable. Comment comprendre autrement ces Indiens venus du Sud de Madagascar dans les années 1930 ? On se rappellera les éléments biographiques qui illustrent ce phénomène dans le roman de V.S. Naipaul $^{22}$, et qui se déroule notamment à Stanleyville, mais aussi au Burundi. Dans les recensements de l'administration belge, nous voyons que la mémoire des origines et des étapes antérieures est solidement conservée. De même la référence à une domiciliation théorique, différente du lieu de naissance, mais aussi de la résidence du moment au Burundi, atteste la permanence de cet horizon élargi.

L'aspect confessionnel intervient de façon décisive dans la structuration des réseaux. Les sources coloniales ne nous aident guère à y voir clair car tous les «Indiens », parfois globalement qualifiés d' "Hindous» dans les dossiers belges, sont traités sur le même pied. Les seules distinctions portent sur les cas des Béloutches et des Goanais, on l'a vu. En recourant à l'onomastique pour déchiffrer les listes de recensement, il est cependant possible de distinguer les Hindous proprement dits des Indiens musulmans et parmi ceux-ci de distinguer les Chiites des Sunnites $^{23}$. Il est plus délicat d'isoler les Ismaéliens parmi les Chiites. Nous avons procédé à un sondage dans les listes de 1936-1939. Sur près de 300 noms disponibles, il apparaît que 75 (soit un quart) seraient hindous, tous à Usumbura. On peut noter que la plupart des artisans (cordonniers, menuisiers, coiffeurs) sont issus de cette catégorie, sans doute dans le cadre de situations de caste. D'autre part, 114, soit un tiers environ, seraient chiites. Notamment tout le petit groupe de Rumonge et Nyanza-Lac est de cette appartenance.

Il faudrait pouvoir croiser les mentions de mariage et d'emploi avec ces appartenances. Les sondages que permettent parfois les sources officielles, déjà citées ${ }^{24}$, des années 1936-1939, vont dans le sens d'une cohérence socioculturelle. Quelques exemples: Amina Molu Visram est l'épouse de Abdul Rasul Alidina Ladak, tous les deux musulmans sunnites; Gokaldas Velji et Vrajdas Makanji Jhakar sont employés chez Juthalal Velji, tous les trois hindous; Labaye est épouse de Karmali Hanseraje, tous les deux hindous; Maryan Bandali Manek est épouse de Gulam Hussen Alidina Ladak, tous les deux chiites; Mohamed Kassim est contremaître chez Hamed Nensi, tous les deux sunnites ; Naganlal est contremaître chez Kanji Virji, tous les deux hindous; Pirsab Hussein Ali est employé de Ali Rawji, tous les deux chiites...

Les seize personnes installées en 1939 à Rumonge et à Nyanza-Lac forment un groupe chiite particulièrement noué ${ }^{25}$. À Rumonge le commerçant Ali Merali a épousé Fatima Bandali, veuve et déjà mère de quatre enfants de son précédent mari (deux fils et deux filles). Lui-même avait aussi deux fils d'un mariage antérieur. Les naissances de ces différents enfants, entre 1920 et 1938, s'étaient étalées

${ }^{22}$ V.S. NaIPaul, À la courbe du fleuve, Paris, Albin Michel, 1982 (traduit de A Bend in the River, New York, A.A. Knopf Inc., 1979).

${ }^{23} \mathrm{Je}$ tiens à remercier vivement Nicole Khouri : sans son aide je serais resté comme aveugle devant ces noms.

${ }^{24}$ Dossiers des Rapports annuels du Ruanda-Urundi, AAB, 149 et 152.

${ }^{25}$ AAB, BUR 221 (Asiatiques, 1939). 
entre Bombay, Chitravad et Rumonge. Le couple semble s'être rencontré à Kigoma avant de s'installer à Rumonge. À Nyanza-Lac, un autre couple, également chiite, travaille pour le grand marchand ismaélien d'Usumbura, Ali Rawji. Un autre commerçant y travaille pour Ali Merali, tous les deux originaires de la région de Bombay.

Dès l'époque allemande les chiites, probablement ismaéliens, semblent avoir été influents, avec les firmes Nasor Virji, Merali Remtulla, Ali Nur Mohamed Kanji.

En résumé, un programme de recherches spécifique, fondé sur des biographies individuelles et familiales, pourrait démêler les jeux de relations et d'exclusion, la géographie et la chronologie des rencontres qui ont ainsi façonné ce milieu dont la complexité n'est abordée qu'indirectement par les sources coloniales.

\section{Les relations ambiguës avec les colonisateurs : utilité économique et méfiances politiques}

Les Indiens occupaient durant la première moitié $\mathrm{du} \mathrm{xx}^{\mathrm{e}}$ siècle une position bien connue, celle d'intermédiaires entre les colonisateurs européens et les colonisés africains, avec tous les quiproquos et les risques que cela impliquait - des risques qui se sont manifestés tragiquement en Ouganda dans les années 1970. Pourtant ces gens, qui ont été volontiers décrits comme des collaborateurs de la colonisation, se trouvaient dans une position historique dont la logique dépassait le seul processus colonial et furent autant appréciés que détestés par les maîtres de l'époque. Ils apparaissaient à la fois comme des avant-postes de la colonisation et comme de possibles agents de subversion. Nous allons donner quelques exemples de cette situation ambiguë sous les dominations successives des Allemands et des Belges.

Les Indiens étaient très appréciés des autorités allemandes pour leur habileté commerciale. Le rapport annuel de 1913 de la Résidence de l’Urundi signale le rôle bénéfique que joue leur sens des affaires : ils ont, y lit-on, réveillé les Arabes trop endormis et ils représentent «des soutiens appréciés du commerce ${ }^{26}$. Cette association de fait autour du souhait commun de voir se développer les échanges et la circulation monétaire (propice à la fiscalité) se traduit par des mesures institutionnelles concrètes, par exemple la mise en place à Usumbura et Gitega de commissions de recours pour l'impôt sur les affaires (Gewerbesteuer) qui vise notamment les commerçants. Dans cette commission on trouve à Usumbura en 1913, le chef du poste von Brandis, un collaborateur de ce dernier, un marchand arabe et deux Indiens, Merali Remtulla et Gulam Husein Ibrahim. On voit cette commission donner satisfaction à une demande d'abattement de la firme Abdallah Alarakia en 1913 et refuser une demande analogue de Nasor Virji en $1914^{27}$.

Dans d'autres circonstances, les rapports sont plus tendus, notamment à propos de l'application des mesures sanitaires liées à la lutte contre la maladie du sommeil

26 Jahresbericht Urundi, op. cit. : 1913.

27 Steuer-Obereinschätzungskommision des Residentur-bezirks Urundi, 1911-1915, AAB, fonds allemands, 116. On apprend du même coup que les profits de ces négociants d'Usumbura allaient de 4000 à 12000 roupies par an. 
sur les rives du lac Tanganyika. La maison Dossa Thawer avait ouvert une duka près du camp de sommeilleux de Nyanza en 1908, mais vu l'inactivité du gérant, le docteur Fischer demande au résident Göring en février 1911 la fermeture de la boutique, ce qui est accordé. Puis la firme ayant fait valoir le coût de son investissement (malgré le rôle des travailleurs du camp dans l'édification de la boutique), le résident von Stegmann autorise la réouverture six mois plus tard (en juillet 1911). Plus grave, plusieurs médecins militaires de la Schlafkrankheitsbekämpfung (SKB) se plaignent en 1910 et 1911 du non-respect des règles sanitaires, interdisant en particulier depuis 1908 la navigation de jour sur le lac. Or Abdallah Alarakia envoie ses agents naviguer de jour d'Ujiji vers Rumonge ou Rumangu, puis vers le Congo, se plaint le docteur Wittrock en décembre 1910. Le résident Göring fait aussitôt fermer la boutique de cette firme à Rumangu. Le même médecin justifie cette mesure en avril 1911 en décrivant le caractère néfaste de ce commerce entre Ujiji, le Sud de l'Urundi et le Congo: selon lui, ce trafic fait circuler la maladie par le biais des porteurs congolais ramenés de la rive belge ${ }^{28}$. La polémique reprendra à ce sujet en juin-juillet 1913 entre les marchands asiatiques d'Ujiji (pétition à l'appui adressée au gouverneur) et les médecins de la $\mathrm{SKB}^{29}$.

Mais un débat plus général se développe en milieu allemand de 1910 à 1913 sur la conduite à tenir à l'égard des Indiens. Le journaliste et pamphlétaire colonial Emil Zimmermann sort un texte virulent en juillet 1910 dans la Deutsche Tageszeitung : selon lui il faut interdire aux Indiens l'accès du Rwanda, future terre de colonisation européenne ${ }^{30}$. Il fait l'éloge des positions à ce sujet de l'ancien responsable du Rwanda et du Burundi, le capitaine von Grawert et de l'ancien gouverneur von Gôtzen, pour dénoncer le laxisme du gouverneur von Rechenberg et du résident du Rwanda, Richard Kandt. Selon lui, ce lobby « libre-échangiste », lié aux Konzern de Hambourg, veut a priori favoriser les Indiens et leurs produits. Il exprime ainsi la nostalgie des mesures prises en mars 1905 et mars 1906 pour faire du Rwanda et du Burundi des secteurs «fermés» (gesperrt) au commerce étranger, qui frappaient en fait surtout les Asiatiques ${ }^{31}$. En décembre 1910, le ministère (le Reichskolonialamt) décide d'arrêter provisoirement toute entrée de nouveaux Indiens dans ces pays ${ }^{32}$.

C'est alors que commence une discussion étonnante entre les autorités locales et le ministère berlinois. En juillet 1911, le résident de l'Urundi von Stegmann informe les négociants de la Deutsch-Ostafrikanische Gesellschaft (DOAG) à Ujiji que

\footnotetext{
${ }^{28}$ Handel,..., op. cit. : 47, I et Verkehr mit Schlafkrankheits-Expeditionen und-Bekämpfung, AAB, fonds allemands, 45, I.

${ }^{29}$ Lettre du gouverneur Schnee, 30 juillet 1913, AAB, fonds allemands, 45, II.

${ }^{30}$ Un mythe de White Highland à la kényane, méconnaissant la réalité démographique locale.

${ }^{31}$ Deutsche Tageszeitung, 28 juillet 1910, AAB, fonds allemands, microfilm 161, 19.

32 Ibid., Reichskolonialamt (RKA, Secrétariat d'État impérial pour les colonies) au gouverneur, arrêté du 14 décembre 1910. Voir J.-P. Chrétien, "La fermeture du Burundi et du Rwanda aux commerçants de l'extérieur (1905-1906). Une décision de l'administration coloniale allemande ", in Laboratoire Connaissance du Tiers-monde, Entreprises et entrepreneurs en Afrique, $19^{e}-20^{e}$ siècles, vol. II, Paris, L'Harmattan, 1983 : 25-47.
} 
seul le Sud du pays reste fermé selon le décret de mars 1906, mais que le Centre et le Nord sont ouverts aux Européens et aux Indiens, contre paiement d'une caution de 250 roupies $^{33}$. Le 28 octobre suivant, le ministre von Lindequist envoie au gouverneur von Rechenberg une lettre circonstanciée qui vise à confirmer l'arrêté restrictif du 14 décembre 1910 à l'encontre des Indiens : il explique que ces commerçants détournent les échanges au profit de "l'Asie britannique », que l'ouverture doit se faire sans eux et qu'il faut plutôt favoriser les Swahili instruits. Donc toute nouvelle entrée d'Indien est interdite. Le principal conseiller du gouverneur, Wilhelm Methner, un excellent connaisseur de la colonie, ne cache pas son désaccord. Le petit commerce indien sert le grand commerce allemand, répond-il le 6 janvier 1912, les firmes O'Swald, Hansing, DOAG en bénéficient, une telle mesure va freiner toute l'économie du Rwanda et du Burundi et il faut laisser en tout cas les firmes déjà installées renouveler leur personnel. Methner prend l'initiative de consulter au préalable les autorités locales. Mais le 10 janvier 1912 le ministre insiste: pas d'accès aux Indiens sans autorisation préalable du RKA, sauf en cas de remplacement de personnel malade ou décédé ${ }^{34}$.

Cependant en mars et avril 1912 les réponses des deux résidents arrivent, celle de Kandt au Rwanda d'abord, puis celle de Langenn au Burundi. Elles sont contradictoires. Kandt insiste sur l'utilité de l'expérience indienne, de leur adaptation à des coûts faibles: "Sans eux, conclut-il, c'est le commerce et les possibilités fiscales du territoire qui seront compromis. " Langenn au contraire affirme qu'un nouvel apport indo-arabo-musulman est peu souhaitable, car ces gens, selon lui, continuent à recourir au troc et, avec eux, l'argent de la Résidence va finir en Inde. Il vaudrait mieux des entreprises allemandes avec des colporteurs noirs pour que l'argent reste chez nous, conclut-il. Dépité et ironique, Methner commente: "une politique de clocher»! Le 20 mai, il doit donc confirmer l'interdiction portée contre l'accès des Indiens dans ces deux pays, mais il rappelle la liste des exceptions (les remplaçants en cas de décès, les agents des Européens, etc. $)^{35}$.

Cependant dès l'année suivante, en 1913, le gouverneur Schnee fait savoir que cet arrêté a été abrogé et que les résidents peuvent apprécier localement. Le 15 juin, il envoie des instructions précises à celui de l'Urundi, à Gitega : l'arrivée prochaine du chemin de fer central à Kigoma, sur le lac Tanganyika, doit entraîner un essor commercial de la région et le RKA. Lui-même a décidé d'autoriser l'accès des Indiens au Rwanda et au Burundi. La Résidence, explique-t-il, peut délivrer toute autorisation en fixant les lieux d'établissement tenant compte des conditions de l'ordre public ${ }^{36}$.

Ge qui est intéressant dans cette affaire, c'est de voir la coalition des éléments militaires (et aussi religieux catholiques) locaux avec les milieux réactionnaires de

${ }_{33}$ Von Stegmann, 7 juillet 1911: Kaufleute und Gewerbetreibende, AAB, fonds allemands, 48, (contenant son décret du ler juillet 1911 sur «l'ouverture du commerce en Urundi»).

34 Tous ces courriers se trouvent dans les liasses 19 et 21, AAB, fonds allemands, microfilm 161.

${ }^{35}$ Lettres de Kandt, ler mars 1912, Kigali, Ibid., liasse 21 ; lettres de Langenn, 10 avril 1912, Usumbura, et réponse de Methner, 20 mai 1912, Dar-es-Salaam, Ibid.

${ }^{36}$ Courrier de Schnee, 15 juin 1913, Dar-es-Salaam, Ibid., liasse 25. 
la métropole contre une vision capitaliste moderne. Les Indiens sont du côté du libre échange, leurs adversaires du côté des valeurs du sang et du sol. Le principal reproche fait aux Indiens est en fait de s'infiltrer en terre allemande au profit des Anglais.

L'époque belge est marquée par les mêmes contradictions entre pragmatisme économique et méfiance politique. Dans ce cas c'est le péril musulman qui est dénoncé. Tout se passe comme si les Hindous étaient invisibles, comme si aucune différence ne pouvait être faite entre les Ismaéliens et les autres et comme si, globalement, tout musulman faisait partie du groupe honni des «arabisés », sentiment répandu depuis les débuts du Congo belge sous influence des missions catholiques $^{37}$. De ce point de vue le rôle néfaste ou l'aveuglement des protestants britanniques est aussi dans la tête de ceux qui cultivent cette méfiance.

Par exemple dans les années 1947-1949 plusieurs signes montrent la crispation des milieux coloniaux à l'égard des Indiens, bien que ce soit aussi en 1948 qu'ait été inaugurée à Usumbura une mosquée ismaélite en présence des autorités belges, d'un représentant de l'Aga Khan et du leader local de cette communauté, le négociant Ali Rawji ${ }^{38}$. En 1948, l'administrateur territorial d'Usumbura se félicite de la diminution sensible des immatriculations d'Asiatiques venus de l'Inde ou de l'East Africa ${ }^{39}$.

Le 30 juin 1949 est signalée au Kivu la venue de «quatre visiteurs indous [sic] dont un religieux musulman originaire de Kampala». Ils ont apporté beaucoup d'argent pour la construction d'une mosquée à Kasongo, n'ont vu ni les autorités, ni les Arabes du lieu, et ils sont repartis en Ouganda par Albertville, Usumbura, puis Kakitumba. Il s'agit de Moulai Mirza Ahmed Hassan, Mohamed Hussein Merali Devji, Karmali Heiji d'Usumbura et Habib Valji de Kampala. Le promoteur financier serait la firme Haji Brothers ${ }^{40}$. Il semble bien que cette délégation de 1949 avait un but strictement religieux dans le cadre de la vie des communautés ismaélites. Mais ce qui obsède l'administration, c'est la possibilité d'une l'infiltration de propagande "communiste » sous le couvert musulman : le gouverneur attire l'attention des administrateurs territoriaux sur ce danger dans une circulaire du 14 juin $1948^{41}$.

La philosophie de cette méfiance est exprimée sans ambages dans le petit livre de Léon Anciaux en 1949². Dénonçant le "péril hindou » en East Africa, dû à l'impéritie des Anglais, et le "grouillement asiatique » de Dar-es-Salaam, «énorme ville hindoue », il rappelle que jadis Kigoma a résonné de «notre jeune et sonore gaieté »(celle des militaires belge) ${ }^{43}$, alors qu'elle est aujourd'hui sous le coup

${ }^{37}$ Voir J.H. Kagabo, L'Islam et les 'Swahili' au Rwanda, Paris, Éditions de l'École des hautes études en sciences sociales, 1988.

${ }^{38}$ Voir les photos de cette cérémonie publiées par Léon Anciaux, Le problème musulman dans l'Afrique belge, Bruxelles, Institut royal colonial belge, 1949, $81 \mathrm{p}$.

${ }^{39}$ Lettre de l'administrateur Leclerc au résident d'Urundi, 26 octobre 1948, AAB, BUR 221/6 («Infiltration musulmane, 1947-49»).

${ }^{40}$ Ibid., courrier de l'administrateur Leclerc, Usumbura, 12 juillet 1949.

${ }^{41}$ Ibid.

${ }^{42}$ L. Anciaux, Le problème musulman..., op. cit.

${ }^{43}$ Kigoma, terminus du chemin de fer allemand sur le lac Tanganyika, à proximité du vieux 
d'« une torpeur morbide », qui contraste avec la prospérité d'Albertville, sur la rive congolaise du lac. On comprend, écrit-il, l'animosité de l'Afrique du Sud vis-à-vis de ces gens de couleur cauteleux ou arrogants. Il redoute de les voir déferler vers la zone de prospérité belge, car, selon lui, la guerre les a enrichis et leurs frais sont inférieurs à ceux des Européens : ils font travailler parents et clients, avec des besoins modiques. Heureusement, conclut-il, le gouverneur Jungers a été vigilant et les visas sont donnés au compte gouttes, vu le danger politique et religieux qu'ils représentent.

Avant l'Égypte de Nasser dans les années 1950, c'est donc l'Inde de Nehru (dont l'influence était censée transiter par l'Est africain britannique) qui semblait inquiétante aux yeux du colonisateur belge. Les Indiens étaient victimes de fantasmes faisant écho à ceux des conservateurs allemands d'avant la Première Guerre mondiale.

Octobre 2007

Jean-Pierre GHRÉTIEN

CNRS, Université Paris 1

Centre d'études des mondes africains

comptoir arabo-swahili d'Ujiji, a été occupé en 1916 par les troupes belges, qui y sont restées jusqu'à ce que, après la guerre, la région soit laissée au Tanganyika britannique. 